Bull. Austral. Math. Soc.

VOL. 58 (1998) [57-73]

\title{
PRIMITIVE IDEALS IN THE COORDINATE RING OF QUANTUM EUCLIDEAN SPACE
}

\author{
SeI-Qwon OH and Chun-Gil Park
}

\begin{abstract}
A twisted group algebra $k^{\sigma} P$ on a free Abelian group $P$ with finite rank and a Poisson structure on $k P$ are studied. As an application, the primitive spectrum of $\mathcal{O}_{q}\left(o k^{n}\right)$, the coordinate ring of quantum Euclidean space, is described and a Poisson algebra $A$ is constructed so that there is a bijection between the primitive spectrum of $\mathcal{O}_{q}\left(o k^{n}\right)$ and the symplectic spectrum of $R$.
\end{abstract}

\section{0 . INTRODUCTION}

The purpose of this paper is to characterise all primitive ideals of $\mathcal{O}_{q}\left(0 k^{n}\right)$, the coordinate ring of quantum Euclidean space and to construct a Poisson algebra $A$ such that there is a natural bijection between the primitive ideals of $\mathcal{O}_{q}\left(o k^{n}\right)$ and the symplectic ideals of $A$, when the ground field $k$ is an uncountably infinite algebraically closed field with characteristic zero and the parameter $q \in k^{*}$ is not a root of unity. This paper confirms S.P. Smith's suggestion for $\mathcal{O}_{q}\left(o k^{n}\right)$; namely that the primitive ideals of certain algebras related to quantum groups should correspond bijectively to the symplectic leaves of a naturally associated Poisson structure on the associated algebraic variety.

In Sections 1 and 2, we establish the structure of the twisted group algebra $k^{\sigma} P$ when $\sigma$ is an antisymmetric bimultiplicative map on the free Abelian group $P$ with finite rank, and the Poisson structure on $k P$ induced by an antisymmetric bilinear map $u$ on $P$. The idea of these sections was given to the author by T.J. Hodges. The authors thank him deeply for permission to use it here. In Section 3 that is a main part of this paper, we characterise the primitive ideals of $\mathcal{O}_{q}\left(o k^{n}\right)$; this arises from the work of Takeuchi [11]. The reader is referred to the articles [10] and [11] for further background of $\mathcal{O}_{q}\left(\mathfrak{o} k^{n}\right)$. The multiplicative rule of this algebra $\mathcal{O}_{q}\left(o k^{n}\right)$ is very similar to that of the quantised Weyl algebra which has been studied by various authors (see $[1,2,4])$, and so the techniques of proofs are similar to those of [1] and [8]. The final section constructs a Poisson algebra $A$ such that there is a bijection between the set of primitive ideals of $\mathcal{O}_{q}\left(o k^{n}\right)$ and the set of symplectic ideals of $B$.

\section{Received 24th November, 1997}

The first author was partially supported by the Basic Science Research Institute Program, Korean Ministry of Education, Project No. BSRI-96-1427.

Copyright Clearance Centre, Inc. Serial-fee code: 0004-9729/98 \$A2.00+0.00. 
Henceforth, we assume throughout that $k$ is an uncountably infinite algebraically closed field with characteristic zero, the parameter $q \in k^{*}$ is not a root of unity and $P$ is a free Abelian group with finite rank unless stated otherwise.

\section{Twisted Group Algebras}

1.1. Since quantum tori are essentially just twisted group algebras (see 1.6), we begin with a brief review of some fairly well-known results about ideals in twisted group algebras.

Let $\sigma \in Z^{2}\left(P, k^{*}\right)$ be a 2-cocycle on a free Abelian group $P$ with finite rank. Then the twisted group algebra $k^{\sigma} P$ is the $k$-algebra with generators $t_{\lambda}$ for $\lambda \in P$ with relations:

$$
t_{\lambda} t_{\mu}=\sigma(\lambda, \mu) t_{\lambda+\mu}
$$

In particular, if $\sigma$ is bimultiplicative and antisymmetric, that is,

$$
\begin{aligned}
\sigma\left(\lambda_{1}+\lambda_{2}, \mu\right) & =\sigma\left(\lambda_{1}, \mu\right) \sigma\left(\lambda_{2}, \mu\right) \\
\sigma(\lambda, \mu) & =\sigma(\mu, \lambda)^{-1},
\end{aligned}
$$

then $\sigma$ is a 2-cocycle on $P$, and thus the twisted group algebra $k^{\sigma} P$ is defined and satisfies the commutation relations:

$$
t_{\lambda} t_{\mu}=\sigma^{2}(\lambda, \mu) t_{\mu} t_{\lambda}
$$

Henceforth, we assume that $\sigma$ is bimultiplicative and antisymmetric on $P$.

1.2. Define

$$
P_{\sigma}=\left\{\lambda \in P \mid \sigma^{2}(\lambda, \mu)=1 \quad \forall \mu \in P\right\} .
$$

Clearly $P_{\sigma}$ is a subgroup of $P$ and free since every subgroup of a free Abelian group (with finite rank) is free.

LEMMA - The centre $Z\left(k^{\sigma} P\right)$ of $k^{\sigma} P$ is $Z\left(k^{\sigma} P\right)=\left\{\sum_{\lambda} a_{\lambda} t_{\lambda} \mid \lambda \in P_{\sigma}\right\}$, which is isomorphic to $k P_{\sigma}$.

Proof: Put $Z=Z\left(k^{\sigma} P\right)$. For $f=\sum_{\lambda} a_{\lambda} t_{\lambda} \in k^{\sigma} P, f \in Z$ if and only if $t_{\mu} f=f t_{\mu}$ for all $\mu \in P$. Since $t_{\mu} f=\sum_{\lambda} \sigma^{2}(\mu, \lambda) a_{\lambda} t_{\lambda} t_{\mu}$, this will occur if and only if $\lambda \in P_{\sigma}$ for all $\lambda$ in the support of $f$.

THEOREM 1.3. There is a bijection preserving inclusions between the ideals of $k^{\sigma} P$ and the ideals of the centre $Z\left(k^{\sigma} P\right)$. That is, if $I$ is an ideal of $k^{\sigma} P$ then $I=\left(I \cap \mathcal{Z}\left(k^{\sigma} P\right)\right) k^{\sigma} P$, and if $J$ is an ideal of $Z\left(k^{\sigma} P\right)$ then $J=J k^{\sigma} P \cap Z\left(k^{\sigma} P\right)$.

Proof: Consider the action of $P$ as automorphisms of $k^{\sigma} P$ defined by

$$
\lambda\left(t_{\mu}\right)=\sigma^{2}(\lambda, \mu) t_{\mu}=t_{\lambda} t_{\mu} t_{\lambda}^{-1}
$$


Let $\mathcal{T}$ be a transversal for $P_{\sigma}$ in $P$. Then the weight space decomposition of $k^{\sigma} P$ under this action is

$$
k^{\sigma} P=\bigoplus_{\nu \in \mathcal{T}} Z\left(k^{\sigma} P\right) t_{\nu}
$$

If $I$ is an ideal of $k^{\sigma} P$ then $I$ must be invariant under this action and so

$$
I=\bigoplus_{\nu} I \cap Z\left(k^{\sigma} P\right) t_{\nu}=\bigoplus_{\nu}\left(I \cap Z\left(k^{\sigma} P\right)\right) t_{\nu}=\left(I \cap Z\left(k^{\sigma} P\right)\right) k^{\sigma} P
$$

If $J$ is an ideal of $Z\left(k^{\sigma} P\right)$ and $x \in J k^{\sigma} P \cap Z\left(k^{\sigma} P\right)$ then $x=\sum_{i} x_{i} f_{i}$ for some $x_{i} \in J$ and $f_{i} \in k^{\sigma} P$. Replace each $f_{i}$ with an element written by the decomposition $\left(^{*}\right)$ and then $x$ can be expressed by $x=\sum_{\nu \in \mathcal{T}} a_{\nu} t_{\nu}$ for some $a_{\nu} \in J$. Since $x \in Z\left(k^{\sigma} P\right)$, if $\nu \notin P_{\sigma}$ then $a_{\nu}=0$ and so $x \in J$. Therefore we have that $J=J k^{\sigma} P \cap Z\left(k^{\sigma} P\right)$.

PROPOSITION 1.4. The centre of the fractional algebra Fract $\left(k^{\sigma} P\right)$ is $\operatorname{Fract}\left(Z\left(k^{\sigma} P\right)\right)$.

Proof: Observe that both $k^{\sigma} P$ and $Z=Z\left(k^{\sigma} P\right)$ are affine domains, thus there exist fractional algebras Fract $\left(k^{\sigma} P\right)$ and Fract $(Z)$. Clearly Fract $(Z)$ is contained in the centre of Fract $\left(k^{\sigma} P\right)$. For $x, y \in k^{\sigma} P$, if $x y^{-1}$ is a central element of Fract $\left(k^{\sigma} P\right)$ then $x y=y x$ and $t_{\lambda} x y^{-1} t_{\lambda}^{-1}=x y^{-1}$ for all $t_{\lambda} \in k^{\sigma} P$, thus we have that $x t_{\lambda} y=y t_{\lambda} x$. Express $y$ as elements of $\left(^{*}\right)$ in the proof of 1.3. Let us call the number of nonzero $z_{\nu} \in Z$ in the expression $y=\sum z_{\nu} t_{\nu}$ the length of $y$. We may assume that $y$ has the shortest length in the set $\left\{y^{\prime} \mid x y^{-1}=x^{\prime} y^{\prime-1}\right.$ for some $\left.x^{\prime}\right\}$. If the length of $y$ is greater than 1 then $0 \neq y-\alpha t_{\lambda} y t_{\lambda}^{-1}$ has shorter length than $y$ for some nonzero scalar $\alpha$ and $t_{\lambda}$ and we have that

$$
x\left(y-\alpha t_{\lambda} y t_{\lambda}^{-1}\right)=x y-\alpha x t_{\lambda} y t_{\lambda}^{-1}=y x-\alpha y t_{\lambda} x t_{\lambda}^{-1}=y\left(x-\alpha t_{\lambda} x t_{\lambda}^{-1}\right) .
$$

Therefore $x y^{-1}=y^{-1} x=\left(x-\alpha t_{\lambda} x t_{\lambda}^{-1}\right)\left(y-\alpha t_{\lambda} y t_{\lambda}^{-1}\right)^{-1}$. This contradicts to the shortest length of $y$, so $y=z_{\nu} t_{\nu}$ and $x y^{-1}=\left(\sigma(\nu, \nu) x t_{-\nu}\right) z_{\nu}^{-1} \in \operatorname{Fract}(Z)$.

Theorem 1.5. Let $\left\{e_{1}, \ldots, e_{n}\right\}$ be a basis of $P$ and let $H$ be the subsemigroup (with identity) of $P$ generated by $e_{1}, \ldots, e_{n}$. Given an antisymmetric bimultiplicative map $\sigma$, let $R$ be a Noetherian $k$-algebra such that $k^{\sigma} H \subseteq R \subseteq k^{\sigma} P$. Then the multiplicative set $\mathcal{C}$ generated by $t_{e_{i}}, i=1, \ldots, n$, is an Ore set of $R$ and the localisation $\mathcal{C}^{-1} R$ is isomorphic to $k^{\sigma} P$. If all prime ideals of $R$ are completely prime then all maximal ideals of $Z\left(k^{\sigma} P\right)$ correspond bijectively to all primitive ideals of $R$ disjoint from $\mathcal{C}$. In fact, the map $M \mapsto\left(M k^{\sigma} P\right)^{c}$ from the maximal ideals of $Z\left(k^{\sigma} P\right)$ into the 
primitive ideals of $R$ disjoint from $\mathcal{C}$ is bijective, where $\left(M k^{\sigma} P\right)^{c}$ is the contraction of $M k^{\sigma} P$.

Proof: Clearly, every element of $\mathcal{C}$ is invertible in $k^{\sigma} P$ and each element of $k^{\sigma} P$ is of the form $b^{-1} a, a \in k^{\sigma} H, b \in \mathcal{C}$, thus the localisation $\mathcal{C}^{-1} R$ is isomorphic to $k^{\sigma} P$ and $\mathcal{C}$ is a left Ore set. Similarly $\mathcal{C}$ is a right Ore set. Note that, by $\mathbf{1 . 3}$, there is a bijection between the set of all maximal ideals of $k^{\sigma} P$ and the set of all maximal ideals of $Z\left(k^{\sigma} P\right)$.

Let $M$ be a maximal ideal of $k^{\sigma} P$. Then the contraction $M^{c}$ to $R$ is a prime ideal disjoint from $\mathcal{C}$ and any prime ideal $Q$ of $R$ properly containing $M^{c}$ contains an element of $\mathcal{C}$ since $M$ is maximal. Since $Q$ is completely prime, $t_{e_{i}} \in Q$ for some $i$ and so $t_{e_{1}} \cdots t_{e_{n}} \in Q \cap \mathcal{C}$. Therefore the intersection of all prime ideals properly containing $M^{c}$ is not equal to $M^{c}$ and so $M^{c}$ is primitive by [7, 9.1.8].

Conversely, let $Q$ be a primitive ideal of $R$ disjoint from $\mathcal{C}$. Then $Q$ is contraction of a prime ideal $M$ of $k^{\sigma} P$. It suffices to show that $M$ is maximal. Let $\lambda_{1}, \ldots, \lambda_{r}$ be a basis of the subgroup $P_{\sigma}$. The elements $t_{\lambda_{i}}, i=1, \ldots, r$, can be written as $t_{\lambda_{i}}=\beta_{i} b_{i}^{-1} a_{i}$ for some $\beta_{i} \in k^{*}, a_{i}, b_{i} \in \mathcal{C}$. Since $t_{\lambda_{i}}$ are central elements of $k^{\sigma} P$ and $Q$ is disjoint from $\mathcal{C}, a_{i}-\alpha_{i} b_{i} \in Q$ for some $\alpha_{i} \in k^{*}$ by [7, 9.1.7]. Hence $M$ contains $t_{\lambda_{i}}-\alpha_{i} \beta_{i}$ for each $i=1, \ldots, r$ and thus $M \cap Z\left(k^{\sigma} P\right)$ is maximal in $Z\left(k^{\sigma} P\right)$ and $M=\left(M \cap Z\left(k^{\sigma} P\right)\right) k^{\sigma} P$ is maximal in $k^{\sigma} P$ by $\mathbf{1 . 3}$.

1.6. (See $[2,2.1],[6]$ and $\left[7,1.5 .10\right.$ (ii)]) Let $\lambda=\left(\lambda_{i j}\right)$ be an $n \times n$ matrix of nonzero elements of $k$ such that $\lambda_{i i}=1$ and $\lambda_{j i}=\lambda_{i j}^{-1}$ for $1 \leqslant i, j \leqslant n$. The multiparameter coordinate ring of quantum affine $n$-space is the $k$-algebra $\mathcal{O}_{\lambda}\left(k^{n}\right)$ generated by elements $x_{1}, \ldots, x_{n}$ subject only to the relations $x_{i} x_{j}=\lambda_{i j} x_{j} x_{i}$ for $1 \leqslant i, j \leqslant n$. Note that $\mathcal{O}_{\lambda}\left(k^{n}\right)$ can be expressed as an $n$-fold iterated skew polynomial ring starting with the field $k$; hence, $\mathcal{O}_{\lambda}\left(k^{n}\right)$ is an affine domain. In particular, if $\lambda_{i j}=q^{-1}, i<j$ then $\mathcal{O}_{\lambda}\left(k^{n}\right)$ is called the coordinate ring of quantum affine $n$-space and denoted $\mathcal{O}_{q}\left(k^{n}\right)$. As in $[2,2.1]$ and [6], we write $P(\lambda)$ for the localisation of $\mathcal{O}_{\lambda}\left(k^{n}\right)$ with respect to the multiplicative set generated by $x_{1}, \ldots, x_{n}$, that is, $P(\lambda)$ is the $k$-algebra generated by $x_{1}, x_{1}^{-1}, \ldots, x_{n}, x_{n}^{-1}$ subject to the relations $x_{i} x_{j}=\lambda_{i j} x_{j} x_{i}$.

Note that $P(\lambda)$ is the twisted group algebra $k^{\sigma} P$, where the free Abelian group $P$ has basis $\left\{e_{1}, \ldots, e_{n}\right\}$ and an antisymmetric bimultiplicative map $\sigma \in Z^{2}\left(P, k^{*}\right)$ is given by

$$
\sigma\left(e_{i}, e_{j}\right)=\lambda_{i j}^{1 / 2}
$$

Conversely, all twisted group algebra $k^{\sigma} P$ with an antisymmetric bimultiplicative map $\sigma$ can be presented as a $P(\lambda)$ for $\lambda=\left(\sigma^{2}\left(e_{i}, e_{j}\right)\right)$. In fact, $\phi: P(\lambda) \longrightarrow k^{\sigma} P$ defined by $\phi\left(x_{i}\right)=t_{e_{i}}$ for all $i=1, \ldots, n$ is an isomorphism. Moreover, the subalgebra $\mathcal{O}_{\lambda}\left(k^{n}\right)$ of $P(\lambda)$ is isomorphic to the twisted semigroup algebra $k^{\sigma} H$, where $H$ is the 
subsemigroup of $P$ generated by $e_{1}, \ldots, e_{n}$.

\section{POISSON TORI}

2.1. Now let $u \in Z^{2}(P, k)$ be an antisymmetric bilinear map. That is,

$$
\begin{aligned}
u\left(\lambda_{1}+\lambda_{2}, \mu\right) & =u\left(\lambda_{1}, \mu\right)+u\left(\lambda_{2}, \mu\right) \\
u(\lambda, \mu) & =-u(\mu, \lambda) .
\end{aligned}
$$

Then it is easily verified that the bracket

$$
\left\{t_{\lambda}, t_{\mu}\right\}=u(\lambda, \mu) t_{\lambda+\mu}
$$

defines a Poisson bracket on the group algebra $k P$. If $k P$ has a Poisson structure then we always assume that it is induced by an antisymmetric bilinear map $u$.

Lemma 2.2. Set

$$
Z_{p}(k P)=\{f \in k P \mid\{f, g\}=0 \forall g \in k P\} .
$$

Then $Z_{p}(k P)=k P_{u}$ where $P_{u}=\{\lambda \in P \mid u(\lambda, \mu)=0 \forall \mu \in P\}$. The Poisson subalgebra $Z_{p}(k P)$ of $k P$, which has the trivial Poisson structure (that is, $\{f, g\}=$ $0 \forall f, g)$, is called the Poisson centre.

Proof: Let $f=\sum_{\lambda} a_{\lambda} t_{\lambda}$. Then $f \in Z_{p}(k P)$ if and only if $\left\{t_{\mu}, f\right\}=0$ for all $\mu \in P$. Since $\left\{t_{\mu}, f\right\}=\sum_{\lambda} u(\mu, \lambda) a_{\lambda} t_{\mu+\lambda}$, this will occur if and only if $\lambda \in P_{u}$ for all $\lambda$ in the support of $f$.

2.3. Recall that a Poisson ideal of a Poisson algebra $A$ is an ideal $I$ such that $\{f, g\} \in I$ for all $f \in I$ and $g \in A$.

Theorem. There is a bijection preserving inclusions between the Poisson ideals of $k P$ and the Poisson ideals of $Z_{p}(k P)$. That is, if $I$ is a Poisson ideal of $k P$ then $I=\left(I \cap Z_{p}(k P)\right) k P$, and if $J$ is a Poisson ideal of $Z_{p}(k P)$ then $J=(J k P) \cap Z_{p}(k P)$.

Proof: Set $Z_{p}=Z_{p}(k P)$. Consider the action of $P$ as linear endomorphisms of $k P$ defined by

$$
\lambda\left(t_{\mu}\right)=u(\lambda, \mu) t_{\mu}=\left\{t_{\lambda}, t_{\mu}\right\} t_{\lambda}^{-1} .
$$

Let $\mathcal{T}$ be a transversal for $P_{u}$ in $P$. Then the weight space decomposition of $k P$ under this action is

$$
k P=\bigoplus_{\nu \in \mathcal{T}} Z_{p} t_{\nu}
$$


If $I$ is a Poisson ideal of $k P$ then $I$ must be invariant under this action and so

$$
I=\bigoplus_{\nu} I \cap Z_{p} t_{\nu}=\bigoplus_{\nu}\left(I \cap Z_{p}\right) t_{\nu}=\left(I \cap Z_{p}\right) k P
$$

If $J$ is a Poisson ideal of $Z_{p}$ and if $x \in(J k P) \cap Z_{p}$ then $x=\sum_{i} x_{i} f_{i}$ for some $x_{i} \in J$ and $f_{i} \in k P$. Replace each $f_{i}$ with an element written by the decomposition $\left({ }^{* *}\right)$ and then $x$ can be expressed by $x=\sum_{\nu \in \mathcal{T}} a_{\nu} t_{\nu}$ for some $a_{\nu} \in J$. Since $x \in Z_{p}$, if $\nu \notin P_{u}$ then $a_{\nu}=0$ and so $x \in J$. Therefore we have that $J=(J k P) \cap Z_{p}$.

2.4. Let $A$ be a Poisson algebra over $k$ and let $Q$ be a prime Poisson ideal of $A$, which means prime in the commutative algebra $A$ and Poisson in $A$. Then the Poisson bracket on $A$ defines a Poisson bracket on Fract $(A / Q)$ and we define $Q$ to be symplectic if

$$
\{a \in \operatorname{Fract}(A / Q) \mid\{a, b\}=0 \forall b \in \operatorname{Fract}(A / Q)\}
$$

reduces to the set of scalars (see [5, A.4.1]). A Poisson algebra $A$ is called symplectic whenever the Poisson ideal $\langle 0\rangle$ is symplectic.

Proposition. The set $Z=\{a \in \operatorname{Fract}(k P) \mid\{a, b\}=0 \forall b \in \operatorname{Fract}(k P)\}$ is equal to the fractional algebra of the Poisson centre $Z_{p}(k P)=k P_{u}$ of $k P$.

Proof: This follows from the modified version of the proof in 1.4. For completeness sake, we write out the proof. Clearly Fract $\left(k P_{u}\right)$ is contained in $Z$. If $x, y$ are elements of $k P$ and $x y^{-1} \in Z$ then $\left\{x, t_{\mu}\right\} y=\left\{y, t_{\mu}\right\} x$ for all $t_{\mu} \in k P$. Express $y$ as elements of $\left(^{* *}\right)$ in the proof of 2.3. Let us call the number of nonzero $z_{\nu} \in k P_{u}$ in the expression $y=\sum z_{\nu} t_{\nu}$ the length of $y$. We may assume that $y$ has the shortest length in the set $\left\{y^{\prime} \mid x y^{-1}=x^{\prime} y^{\prime-1}\right.$ for some $\left.x^{\prime}\right\}$. If the length of $y$ is greater than 1 then $0 \neq y-\alpha\left\{y, t_{\mu}\right\} t_{\mu}^{-1}$ has shorter length than $y$ for some scalar $\alpha \in k^{*}$ and $t_{\mu} \in k P$, and

$$
x\left(y-\alpha\left\{y, t_{\mu}\right\} t_{\mu}^{-1}\right)=y x-\alpha x\left\{y, t_{\mu}\right\} t_{\mu}^{-1}=y\left(x-\alpha\left\{x, t_{\mu}\right\} t_{\mu}^{-1}\right) .
$$

Therefore $x y^{-1}=\left(x-\alpha\left\{x, t_{\mu}\right\} t_{\mu}^{-1}\right)\left(y-\alpha\left\{y, t_{\mu}\right\} t_{\mu}^{-1}\right)^{-1}$, which is a contradiction to the shortest length of $y$. Hence we have that $y=z_{\nu} t_{\nu}$ and $x y^{-1}=\left(x t_{-\nu}\right) z_{\nu}^{-1} \in$ Fract $\left(k P_{u}\right)$.

THEOREM 2.5. Let $\left\{e_{1}, \ldots, e_{n}\right\}$ be a basis of $P$ and let $H$ be the subsemigroup (with identity) of $P$ generated by $\left\{e_{1}, \ldots, e_{n}\right\}$. Let $k P$ be the Poisson algebra induced by an antisymmetric bilinear map $u$, let $A$ be a sub-Poisson and Noetherian subalgebra such that $k H \subseteq A \subseteq k P$ and let $\mathcal{C}$ be the multiplicative set generated by $t_{e_{1}}, \ldots, t_{e_{n}}$. Then $\mathcal{C}^{-1} A=k P$ and extensions of all symplectic ideals of $A$ disjoint from $\mathcal{C}$ are maximal Poisson ideals of $k P$. 
Proof: Clearly, the localisation $\mathcal{C}^{-1} A$ is isomorphic to $k P$. Let $Q$ be a symplectic ideal of $A$ disjoint from $\mathcal{C}$ and let $\lambda_{1}, \ldots, \lambda_{r}$ be a basis of the subgroup $P_{u}$. Then $Q$ is contraction of a Poisson ideal $M$ of $k P$ and $t_{\lambda_{i}}=a_{i} b_{i}^{-1}$ for some $a_{i}, b_{i} \in \mathcal{C}$, $i=1, \ldots, r$, and so $a_{i}-\alpha_{i} b_{i} \in Q$ for some $\alpha_{i} \in k^{*}$. Hence $t_{\lambda_{i}}-\alpha_{i} \in M \cap Z_{p}(k P)$. Therefore $M$ is a maximal Poisson ideal of $k P$ by 2.3 .

Corollary 2.6. Under the same conditions as 2.5, let $P=P_{u} \oplus P^{\prime}$ for some subgroup $P^{\prime}$. Then there is a bijection between the set of symplectic ideals of $A$ and the set of maximal Poisson ideals of $k P$.

Proof: Let $M$ be a maximal Poisson ideal of $k P$ and let $e_{1}, \ldots, e_{r}$ be a basis of $P_{u}$. Then $M=\left\langle t_{e_{1}}-\alpha_{1}, \ldots, t_{e_{r}}-\alpha_{r}\right\rangle k P$ for some $\alpha_{i} \in k^{*}$ by 2.3. Let us prove that the contraction $M^{c}$ to $A$ is symplectic. Clearly, $M^{c}$ is a prime ideal of $A$ since $M$ is prime in $k P$. Note that the antisymmetric bilinear map $u^{\prime}=\left.u\right|_{P^{\prime} \times P^{\prime}}$ gives a Poisson structure on $k P^{\prime}$ and $P_{u^{\prime}}^{\prime}$ is trivial. Since $k P^{\prime} \cong k P / M=\overline{\mathcal{C}}\left(A / M^{c}\right)$ is symplectic by 2.4 and $\operatorname{Fract}\left(A / M^{c}\right)$ is isomorphic to $\operatorname{Fract}\left(k P^{\prime}\right), M^{c}$ is a symplectic ideal of $A$. Hence the conclusion follows from $\mathbf{2 . 5}$.

LEMMA 2.7. Given an antisymmetric bimultiplicative map $\sigma \in Z^{2}\left(P, k^{*}\right)$ and $0 \neq q \in k$ which is not a root of unity, define $u: P \times P \longrightarrow k$ by

$$
\sigma(\lambda, \mu)=q^{u(\lambda, \mu)} \quad \forall \lambda, \mu \in P .
$$

Then $u$ is an antisymmetric bilinear map and

$$
Z\left(k^{\sigma} P\right) \cong Z_{p}(k P)=k P_{u}
$$

Proof: Clearly, $u$ is an antisymmetric bilinear map and $P_{\sigma}=P_{u}$ since $q$ is not a root of unity, and so $Z\left(k^{\sigma} P\right) \cong Z_{p}(k P)=k P_{u}$ by $\mathbf{1 . 2}$ and $\mathbf{2 . 2}$.

\section{Primitive ideals IN-THE COORDinate RING OF QUANTUM EuClidean SPACE}

Definition 3.1: (See $\left[\mathbf{9}, \mathbf{5}, \mathbf{1 0}\right.$, Section 4] and $[\mathbf{1 1}, 5.1]$ ) Let $q \in k^{*}$. For each positive integer $n$, the coordinate ring of quantum Euclidean space $\mathcal{O}_{q}\left(\mathfrak{o} k^{2 n}\right)$ is the $k$-algebra generated by $2 n$ variables $y_{1}, x_{1}, y_{2}, x_{2}, \cdots, y_{n}, x_{n}$ satisfying the following relations:

$$
\begin{aligned}
y_{i} y_{j} & =q y_{j} y_{i} & & (i<j) \\
x_{i} y_{j} & =q y_{j} x_{i} & & (i \neq j) \\
x_{j} x_{i} & =q x_{i} x_{j} & & (i<j) \\
x_{j} y_{j} & =y_{j} x_{j}+\left(1-q^{2}\right) \sum_{1 \leqslant l<j} q^{l-j} y_{l} x_{l} & & (\text { all } j) .
\end{aligned}
$$


The coordinate ring of quantum Euclidean space $\mathcal{O}_{q}\left(0 k^{2 n+1}\right)$ is the $k$-algebra generated by $2 n+1$ variables $z_{0}, y_{1}, x_{1}, y_{2}, x_{2}, \cdots, y_{n}, x_{n}$ satisfying the following relations:

$$
\begin{aligned}
z_{0} y_{j} & =q y_{j} z_{0} & & (\text { all } j) \\
z_{0} x_{j} & =q^{-1} x_{j} z_{0} & & (\text { all } j) \\
y_{i} y_{j} & =q y_{j} y_{i} & & (i<j) \\
x_{i} y_{j} & =q y_{j} x_{i} & & (i \neq j) \\
x_{j} x_{i} & =q x_{i} x_{j} & & (i<j) \\
x_{j} y_{j} & =y_{j} x_{j}+\left(1-q^{2}\right) \sum_{1 \leqslant l<j} q^{l-j} y_{l} x_{l}+q^{(1 / 2)-j}(1-q) z_{0}^{2} & & (\text { all } j) .
\end{aligned}
$$

Hereafter, we write $\mathcal{O}_{q}^{n}$ for $\mathcal{O}_{q}\left(\mathfrak{o} k^{2 n}\right)$.

LEmma 3.2. The algebra $\mathcal{O}_{q}^{n}$ is a Noetherian domain and all its prime ideals are completely prime.

Proof: By $[\mathbf{9}, \mathbf{5}], \mathcal{O}_{q}^{n}$ is an iterated skew polynomial ring

$$
\mathcal{O}_{q}^{n}=k\left[y_{1}\right]\left[x_{1} ; \beta_{1}\right]\left[y_{2} ; \alpha_{2}\right]\left[x_{2} ; \beta_{2}, \delta_{2}\right] \cdots\left[y_{n} ; \alpha_{n}\right]\left[x_{n} ; \beta_{n}, \delta_{n}\right]
$$

for certain automorphisms $\alpha_{i}$ and $\beta_{i}$. Thus, it is a Noetherian domain. Moreover it is easy to check that $\alpha_{i}, \beta_{i}$ and left $\beta_{i}$-derivation $\delta_{i}$ satisfy the condition of $[3,2.3]$, and so all prime ideals of $\mathcal{O}_{q}^{n}$ are completely prime.

LEMMA 3.3. In $\mathcal{O}_{q}^{n}$, set

$$
z_{i}=q^{-2} x_{i} y_{i}-y_{i} x_{i}=q^{-2}\left(1-q^{2}\right) \sum_{1 \leqslant l \leqslant i} q^{l-i} y_{l} x_{l}
$$

for $i=1, \ldots, n$. Then $z_{n}$ is central, all $z_{i}$ are normal and $y_{i}, y_{i-1}, x_{i}, x_{i-1}$ are normal modulo $z_{i-1}$ for each $i \geqslant 1 \quad\left(z_{0}=0\right)$. More precisely,

$$
\begin{aligned}
& z_{j} y_{i}=y_{i} z_{j} \\
& z_{j} x_{i}=x_{i} z_{j} \\
& (i \leqslant j) \\
& z_{j} y_{i}=q^{2} y_{i} z_{j} \\
& z_{j} x_{i}=q^{-2} x_{i} z_{j} \\
& (i>j) \\
& q^{2} z_{i}=x_{i} y_{i}-q^{2} y_{i} x_{i} \\
& q z_{i}=x_{i+1} y_{i+1}-y_{i+1} x_{i+1} \quad(i \geqslant 1) \\
& q^{2} z_{i}=\left(1-q^{2}\right) y_{i} x_{i}+q z_{i-1} \\
& z_{j} z_{i}=z_{i} z_{j}
\end{aligned}
$$

PROOF: This follows immediately from direct calculations.

Definition 3.4: (See $[8,1.4])$ Let $\wp_{n}=\left\{z_{1}, y_{1}, x_{1}, z_{2}, y_{2}, x_{2}, \ldots, z_{n}, y_{1}, x_{n}\right\}$ be a subset of $\mathcal{O}_{q}^{n}$. We shall say that $T \subseteq \wp_{n}$ is admissible if $T$ satisfies the conditions:

(i) $y_{i} \in T$ or $x_{i} \in T$ if and only if $z_{i} \in T$ and $z_{i-1} \in T, 2 \leqslant i \leqslant n$.

(ii) $y_{1} \in T$ or $x_{1} \in T$ if and only if $z_{1} \in T$. 
The definition of an admissible set should be compared with that of a $p$-sequence in $[1,4.2]$. In fact, if $T$ is an admissible set then

$$
S=T-\left\{z_{i} \mid y_{i} \in T \text { or } x_{i} \in T\right\}
$$

is $p$-sequence in $\mathcal{O}_{q}^{n}$. Note that the ideal generated by $T$ is equal to the ideal generated by the $p$-sequence $S$ as in $[1,4.3]$.

LEMMA 3.5. For each prime ideal $P$ of $\mathcal{O}_{q}^{n}, P \cap \wp_{n}$ is an admissible set.

ProOF: This follows immediately from 3.3 .

LEMмA 3.6. Let $T$ be an admissible set. Then the ideal $\langle T\rangle$ is completely prime and there is a subalgebra $A_{T}$ of $\mathcal{O}_{q}^{n}$ such that $A_{T}$ is a multiparameter coordinate ring of quantum affine space $\mathcal{O}_{\lambda_{T}}\left(k^{m}\right)$ for some matrix $\lambda_{T}=\left(\lambda_{i j}\right), \lambda_{i j}=1, q^{ \pm 1}$ or $q^{ \pm 2}$ and

$$
A_{T}=\mathcal{O}_{\lambda_{T}}\left(k^{m}\right) \subseteq \mathcal{O}_{q}^{n} /\langle T\rangle \subseteq P\left(\lambda_{T}\right)
$$

Proof: The ideal $\langle T\rangle$ is completely prime as in $[\mathbf{1}, 4.5]$. Put

$$
\begin{aligned}
& T_{y}=\left\{y_{j} \mid y_{j} \notin T, x_{j} \in T\right\} \\
& T_{x}=\left\{x_{j} \mid x_{j} \notin T, y_{j} \in T\right\} \\
& S_{T}=\left\{y_{j}, z_{j} \mid z_{j} \notin T\right\} \cup\left\{y_{j} \mid z_{j} \in T, y_{j} \notin T, x_{j} \notin T\right\} \cup T_{y} \cup T_{x},
\end{aligned}
$$

and let $A_{T}$ be the subalgebra of $\mathcal{O}_{q}^{n}$ generated by all elements of $S_{T}$ and let $m$ be the number of elements in $S_{T}$. Since there is no index $i$ such that both $y_{i}$ and $x_{i}$ are in $S_{T}$, we have that, by 3.1 and $3.3, s_{i} s_{j}=\lambda_{i j} s_{j} s_{i}$ for any pair $s_{i}, s_{j} \in S_{T}$, and so $A_{T^{\prime}}=\mathcal{O}_{\lambda_{T}}\left(k^{m}\right)$ for the $m \times m$-matrix $\lambda_{T}=\left(\lambda_{i j}\right)$ by 1.6. Since

$$
T-\left\{z_{i} \mid y_{i} \in T \text { or } x_{i} \in T\right\}
$$

is a normalising sequence of generators for $\langle T\rangle$ and each element of $S_{T}$ is not in the ideal $\langle T\rangle$, we get immediately that $A_{T} \cap\langle T\rangle=0$, hence $A_{T}$ is embedded into $\mathcal{O}_{q}^{n} /\langle T\rangle$. The image in $\mathcal{O}_{q}^{n} /\langle T\rangle$ of the multiplicative set generated by all the elements in $S_{T}$ is a right and left Ore set in $\mathcal{O}_{q}^{n} /\langle T\rangle$ as in $[1,4.8]$. Let $B_{T}$ denote the localisation of $\mathcal{O}_{q}^{n} /\langle T\rangle$ at this set. Since $\left(1-q^{2}\right) y_{i} x_{i}=q^{2} z_{i}-q z_{i-1}\left(z_{0}=0\right)$ by 3.3 and all nonzero generators $\bar{y}_{j} \in B_{T}$ are invertible, we have that all $\bar{x}_{j} \in \mathcal{O}_{q}^{n} /\langle T\rangle$ are in $B_{T}$. Therefore,

$$
A_{T}=\mathcal{O}_{\lambda_{T}}\left(k^{m}\right) \subseteq \mathcal{O}_{q}^{n} /\langle T\rangle \subseteq B_{T}=P\left(\lambda_{T}\right)
$$

It is cumbersome to use the standard overlining notation for images in factor rings of $\mathcal{O}_{q}^{n}$ and so we shall write, for example, $x_{i}$ for the image of $x_{i}$ in a factor ring if no confusion arises.

3.7. Let $T$ be an admissible set such that $y_{i} \in T$ and $x_{i} \in T$ for some $i$. Then the index $i$ is said to be removable in $T$. 
LEMмA. If $T$ is an admissible set of $\mathcal{O}_{q}^{n}$ with removable indices then there is an integer $m<n$ and an admissible set $T^{\prime}$ of $\mathcal{O}_{q}^{m}$ such that $\mathcal{O}_{q}^{n} /\langle T\rangle \cong \mathcal{O}_{q}^{m} /\left\langle T^{\prime}\right\rangle$ and $T^{\prime}$ has no removable indices.

Proof: Suppose that $j$ is removable in $T$. Then there is a natural epimorphism $\phi$ from $\mathcal{O}_{q}^{n-1}$ onto $\mathcal{O}_{q}^{n} /\langle T\rangle$ given by

$$
\begin{array}{llrl}
y_{i} \mapsto q^{-1} y_{i}, & x_{i} \mapsto x_{i}, & i<j \\
y_{i} \mapsto y_{i+1}, & x_{i} \mapsto x_{i+1}, & i \geqslant j .
\end{array}
$$

Since $\operatorname{ker}(\phi)$ is prime by 3.6, $\operatorname{ker}(\phi) \cap \wp_{n-1}$ is an admissible set of $\mathcal{O}_{q}^{n-1}$ by 3.5. An induction on $n$ completes the proof.

3.8. From here to 3.11 , we shall work to find the centre of $P\left(\lambda_{T}\right)=B_{T}$ in $\mathbf{3 . 6}$ when $T$ has no removable indices.

Let $T$ be an admissible set of $\mathcal{O}_{q}^{n}$ without removable indices. Note that $\left(q^{-2}-1\right) y_{1} x_{1}^{-1}=z_{1}^{-1} y_{1}^{2}$ and $z_{n}$ are central elements of Fract $\mathcal{O}_{q}^{n}$. For $S_{T}$ as in the proof of 3.6, put

$$
\begin{array}{llll}
U_{T}=S_{T}-\left\{z_{1}, z_{n}\right\} & c_{-1}=z_{1}^{-1} y_{1}^{2} & c_{0}=z_{n} & \text { if } z_{n} \notin T \text { and } z_{1} \notin T \\
U_{T}=S_{T}-\left\{z_{1}\right\} & c_{-1}=z_{1}^{-1} y_{1}^{2} & c_{0}=0 & \text { if } z_{n} \in T \text { and } z_{1} \notin T \\
U_{T}=S_{T}-\left\{z_{n}\right\} & c_{-1}=0 & c_{0}=z_{n} & \text { if } z_{n} \notin T \text { and } z_{1} \in T \\
U_{T}=S_{T} & c_{-1}=0 & c_{0}=0 & \text { if } z_{n} \in T \text { and } z_{1} \in T .
\end{array}
$$

\section{LEMMA .}

$$
\text { ind } \begin{aligned}
U_{T}: & =\left\{i \mid z_{i} \notin U_{T}\right\} \\
& =\left\{i_{1}, i_{1}+1, i_{1}+2, \cdots, i_{1}+v_{1}\right\} \cup\left\{i_{2}, i_{2}+1, i_{2}+2, \cdots, i_{2}+v_{2}\right\} \\
\cup & \cdots \cup\left\{i_{r}, i_{r}+1, i_{r}+2, \cdots, i_{r}+v_{r}\right\}
\end{aligned}
$$

for some nonnegative integers $v_{i}$ and positive integers $1=i_{1}<i_{2}<\cdots<i_{r}$ satisfying $i_{j}-\left(i_{j-1}+v_{j-1}\right) \geqslant 2, i_{r}+v_{r}=n$.

Proof: Since $T$ has no removable indices, it follows immediately from the definition of admissible set.

LEMMA 3.9. Let $T, U_{T}, i_{j}$ and $v_{j}$ be as in 3.8 .

(1) Let $v_{r}$ be odd. Rewrite the elements of

$$
\left(U_{T} \cap\left\{y_{i_{r}}, x_{i_{r}}, y_{i_{r}+1}, x_{i_{r}+1}, \ldots, y_{n}, x_{n}\right\}\right) \cup\left\{z_{i_{r}-1}\right\}
$$

as $u_{1}, u_{2}, \ldots, u_{p}$, say, where $u_{1}>u_{2}>\ldots>u_{p}$ in the ordering

$$
y_{n}>y_{n-1}>\ldots>y_{1}>z_{1}>x_{1}>z_{2}>x_{2}>\ldots>z_{n}>x_{n} .
$$


Note that $p$ is odd. Then

$$
c_{r}=u_{1}^{\varepsilon_{1}} u_{2}^{-\varepsilon_{2}} \cdots u_{p-1}^{-\varepsilon_{p-1}} u_{p}^{\varepsilon_{p}}, \quad \varepsilon_{k}= \begin{cases}2 & u_{k} \neq z_{i_{r}-1} \\ 1 & u_{k}=z_{i_{r}-1}\end{cases}
$$

is a central element of $B_{T}$. If $v_{r}$ is even then put $c_{r}=0$.

(2) Let $v_{j}, 1<j<r$, be even. Rewrite the elements of

$$
\left(U_{T} \cap\left\{y_{i_{j}}, x_{i_{j}}, y_{i_{j}+1}, x_{i_{j}+1}, \ldots, y_{i_{j}+v_{j}}, x_{i_{j}+v_{j}}\right\}\right) \cup\left\{z_{i_{j}-1}\right\}
$$

as $u_{1}, u_{2}, \ldots, u_{p}$, say, where $u_{1}>u_{2}>\ldots>u_{p}$ in the ordering

$$
y_{n}>y_{n-1}>\ldots>y_{1}>z_{1}>x_{1}>z_{2}>x_{2}>\ldots>z_{n}>x_{n} .
$$

Note that $p$ is even. Then

$$
c_{j}=u_{1}^{\varepsilon_{1}} u_{2}^{-\varepsilon_{2}} \cdots u_{p-1}^{\varepsilon_{p-1}} u_{p}^{-\varepsilon_{p}}, \quad \varepsilon_{k}=\left\{\begin{array}{cc}
2 & u_{k} \neq z_{i_{j}-1} \\
1 & u_{k}=z_{i_{j}-1}
\end{array}\right.
$$

is a central element of $B_{T}$. If $v_{j}$ is odd then put $c_{j}=0$.

Proof: This follows by direct calculations using $\mathbf{3 . 1}$ and 3.3.

Lemma 3.10. Let $T, U_{T}, i_{j}, v_{j}$ and $c_{i}$ be as 3.8 and 3.9. Put

$$
V_{T}=U_{T}-\left\{z_{i_{j}-1} \mid c_{j} \neq 0, j=2, \ldots, r\right\}
$$

(1) Let $v_{1}$ be odd and let $V_{T} \cap\left\{z_{1}, z_{2}, \ldots, z_{n}\right\} \neq \emptyset$. Assume that $i$ is the least index such that $z_{i} \in V_{T}$. Rewrite the elements of $\left(V_{T} \cap\left\{y_{1}, x_{1}, \ldots, y_{i-1}, x_{i-1}\right\}\right) \cup\left\{z_{i}\right\}$ as $u_{1}, u_{2}, \ldots, u_{p}$, say, where $u_{1}>u_{2}>\ldots>u_{p}$ in the ordering

$$
y_{n}>y_{n-1}>\ldots>y_{1}>z_{1}>x_{1}>z_{2}>x_{2}>\ldots>z_{n}>x_{n}
$$

Note that $p$ is odd. Then

$$
c_{1}=u_{1}^{\varepsilon_{1}} u_{2}^{-\varepsilon_{2}} \cdots u_{p-1}^{-\varepsilon_{p-1}} u_{p}^{\varepsilon_{p}}, \quad \varepsilon_{k}=\left\{\begin{array}{cc}
2 & u_{k} \neq z_{i} \\
1 & u_{k}=z_{i}
\end{array}\right.
$$

is a central element of $B_{T}$. Put $z=z_{i}$.

(2) Let $v_{1}$ be odd and let $V_{T} \cap\left\{z_{1}, z_{2}, \ldots, z_{n}\right\}=\emptyset$. Rewrite the elements of $V_{T} \cap\left\{y_{1}, x_{1}, \ldots, y_{n}, x_{n}\right\}$ as $u_{1}, u_{2}, \ldots, u_{p}$, say, where $u_{1}>u_{2}>\ldots>u_{p}$ in the ordering

$$
y_{n}>y_{n-1}>\ldots>y_{1}>z_{1}>x_{1}>z_{2}>x_{2}>\ldots>z_{n}>x_{n}
$$


Note that $p$ is odd. Then

$$
c_{1}=u_{1} u_{2}^{-1} \cdots u_{p-1}^{-1} u_{p}
$$

is a central element of $B_{T}$. Put $z=\left\{\begin{array}{ll}y_{1} & y_{1} \in V_{T} \\ x_{1} & x_{1} \in V_{T} .\end{array}\right.$ If $v_{1}$ is even then put $c_{1}=0$.

Proof: As in the proof of 3.11, this follows by 3.1 and $\mathbf{3 . 3}$.

Lemma 3.11. Let $B_{T}$ be as in 3.6 and let $T, V_{T}, c_{1}$ and $z$ be as in 3.10. Put

$$
\begin{array}{ll}
W_{T}=V_{T}-\{z\} & c_{1} \neq 0 \\
W_{T}=V_{T} & c_{1}=0 .
\end{array}
$$

Then the centre of $B_{T}$ is the subalgebra generated by $\left\{c_{i}^{ \pm 1} \mid c_{i} \neq 0\right.$, for $i=$ $-1,0, \ldots, r\}$. If $B_{T}$ is presented by $B_{T}=k^{\sigma} P$ (see 1.6) then $P=P^{\prime} \oplus P_{\sigma}$ and the rank of $P_{\sigma}$ is equal to the number of nonzero $c_{i}$ 's, $i=-1,0, \ldots, r$.

Proof: Let $S_{T}$ be as in the proof of 3.6. Note that each element of $S_{T}-W_{T}$ is the divisor $s$ of nonzero $c_{i}, i=-1,0,1, \ldots, r$, such that the power of $s$ is 1 or -1 , for example, if $c_{-1} \neq 0$ then $z_{1} \in S_{T}-W_{T}$ and if $c_{r} \neq 0$ then $z_{i_{r}-1} \in S_{T}-W_{T}$, and that the number of elements in $W_{T}$ is even. Rewrite the elements of $W_{T}$ as $w_{1}, w_{2}, \ldots, w_{2 p}$, say, where $w_{1}>w_{2}>\ldots>w_{2 p}$ in the ordering

$$
x_{n}>z_{n}>y_{n}>x_{n-1}>z_{n-1}>y_{n-1}>\ldots>x_{1}>z_{1}>y_{1} .
$$

By the McConnell-Pettit criterion [6], we see that the subalgebra $W$ of $B_{T}$ generated by all $w_{i}^{ \pm 1}$ is simple. But in 3.13, we shall give another proof for the simplicity of $W$ in order to avoid routine and messy calculations in finding the determinant of a huge matrix. Since the subalgebra of $B_{T}$ generated by $\left\{c_{i}^{ \pm 1} \mid c_{i} \neq 0\right\}$ is contained in the centre of $B_{T}$ and each element $s \in S_{T}-W_{T}$ is a divisor of each the nonzero $c_{i}$ with power 1 , we have that the centre of $B_{T}$ is the subalgebra generated by the nonzero $c_{i}^{ \pm 1}$ and $P=P^{\prime} \oplus P_{\sigma}$, where $P^{\prime}$ and $P_{\sigma}$ are the subgroups corresponding $W$ and the centre $Z\left(B_{T}\right)$, respectively.

Proposition 3.12. Let $A$ be a simple algebra over a field $k$ and let $B=$ $A[y ; \alpha][x ; \beta]$ be an iterated skew polynomial ring, where

$$
\alpha: A \longrightarrow A, \quad \beta: A[y ; \alpha] \longrightarrow A[y ; \alpha]
$$

are automorphisms such that $\beta(A)=A, \beta(y)=d y, d \in k^{*}$ and for each pair $i, j$ of nonnegative integers with $i+j \geqslant 1$, there is no $0 \neq a \in A$ satisfying the two conditions

$$
d^{j} \alpha(a)=a, \quad d^{-i} \beta(a)=a .
$$


Then the localisation $C=A\left[y^{ \pm 1}, x^{ \pm 1}\right]$ at the multiplicative set generated by $y$ and $x$ is simple.

Proof: Note that all elements of $C$ are uniquely expressed in a form $f=$ $\sum a_{i j} y^{i} x^{j}$. Let us denote by length of $f$ the number of nonzero $a_{i j} \in A$. For a nonzero ideal $I$ of $C$, choose $0 \neq f \in I$ with the smallest length. Suppose that the length of $f$ is greater than 1 . We may assume that $f$ is of the form $f=a+b y^{i} x^{j}+$ (other terms) for some nonzero $a, b \in A$ and some nonnegative integers $i, j$ with $i+j \geqslant 1$. Since $A b\left(\alpha^{i} \beta^{j}(A)\right)=A$, we may also assume that $b=1$. By our hypothesis, we have that $d^{j} \alpha(a) \neq a$ or $d^{-i} \beta(a) \neq a$, say $d^{j} \alpha(a) \neq a$. Then

$$
f y-d^{j} y f=\left(a-d^{j} \alpha(a)\right) y+\text { (other terms) } \neq 0
$$

and the length of $f y-d^{j} y f \in I$ is less than that of $f$. This is a contradiction. Hence the length of $f$ is 1 and so $f$ is invertible.

Corollary 3.13. Let $A, B, C$, and $\alpha, \beta$ be as in $\mathbf{3 . 1 2}$ and let $d \in k^{*}$ not be a root of unity. If $\beta=\alpha^{r}$ or $\alpha=\beta^{r}$ in $A$ for some $r \geqslant 1$ then $C$ is simple.

Proof: For some pair $i, j$ of nonnegative integers and $i+j \geqslant 1$, and some nonzero $a \in A$, suppose that

$$
d^{j} \alpha(a)=a, \quad d^{-i} \beta(a)=a .
$$

Suppose that $\beta=\alpha^{r}$. Then $a=d^{-i} \beta(a)=d^{-i} \alpha^{r}(a)=d^{-i-r j} a$, which is absurd because $d$ is not a root of unity and $-i-r j<0$. Hence $C$ is simple by 3.12. For the case $\alpha=\beta^{r}$, the proof is similar.

(Proof for the simplicity of $W$ in the proof of 3.11.) Under the same notations as in the proof of 3.11, note that $w_{2 i-1} w_{2 i}=q^{k} w_{2 i} w_{2 i-1}$ for some $k= \pm 1$ or \pm 2 because $y_{j}$ and $z_{j}$ with the same index $j$ cannot be $w_{2 i-1}=z_{j}, w_{2 i}=y_{j}$ by the construction of $W_{T}$. Then, by induction on $p$, the simplicity of $W$ follows immediately from 3.13. [

3.14. Let $T$ be an admissible set $\mathcal{O}_{q}^{n}$ without removable indices. Call the rank of $P_{\sigma}$ in 3.11 the degree of $T$, and denote it by $\operatorname{deg}(\mathrm{T})$.

If $T$ is arbitrary admissible set of $\mathcal{O}_{q}^{n}$ then there are $m \leqslant n$ and an admissible set $T^{\prime}$ of $\mathcal{O}_{q}^{m}$ without removable indices by 3.7. Denote $\operatorname{deg}(T)=\operatorname{deg}\left(T^{\prime}\right)$. Call an admissible set $T$ connected if $T$ satisfies the property: if $z_{i} \in T, z_{j} \in T$ and $i<j$ then $z_{l} \in T$ for all $i \leqslant l \leqslant j$ (see $[8,1.6(2)]$ ). Clearly every admissible set $T^{\prime}$ without removable indices is the disjoint union of connected admissible subsets without removable indices. By $3.9,3.10$ and 3.11 , it is easy to find $\operatorname{deg}(T)$ for any admissible set $T$ without removable indices.

Theorem. Let $T$ be an admissible set of $\mathcal{O}_{q}^{n}$ and let $\operatorname{Prim}_{T}\left(\mathcal{O}_{q}^{n}\right)$ be the set of all primitive ideals $P$ of $\mathcal{O}_{q}^{n}$ such that $P \cap \wp_{n}=T$. Then there is a bijection between 
$\operatorname{Prim}_{T}\left(\mathcal{O}_{q}^{n}\right)$ and the set of all maximal ideals $\operatorname{Max}\left(k\left[t_{1}^{ \pm 1}, \ldots, t_{s}^{ \pm 1}\right]\right)$, where $s=\operatorname{deg} T$, and $\operatorname{Prim}\left(\mathcal{O}_{q}^{n}\right)=\bigsqcup_{T} \operatorname{Prim}_{T}\left(\mathcal{O}_{q}^{n}\right)$.

Proof: If $n=1$ then $\mathcal{O}_{q}^{1}$ is the commutative polynomial ring with two variables, hence the theorem follows from Hilbert's Nullstelnsatz because every primitive ideal of a commutative ring is maximal. Because of induction on $n$, and $\mathbf{3 . 7}$, we may assume that $T$ has no removable indices. The theorem then follows immediately from 1.5, 3.2, 3.6 and 3.11 .

\section{Poisson strcture of THE Quantum EuClidean SPaCe}

4.1. In 3.6, if $T=\emptyset$ then the subalgebra $A_{\emptyset}$ of $\mathcal{O}_{q}^{n}$ is generated by

$$
S_{\emptyset}=\left\{y_{1}, \ldots, y_{n}, z_{1}, \ldots, z_{n}\right\}
$$

and thus the matrix $\lambda_{\emptyset}$ is

$$
\lambda_{\emptyset}=\left(\lambda_{i j}\right)=\left(\begin{array}{cccccccccc}
1 & q & q & \cdots & q & 1 & 1 & 1 & \cdots & 1 \\
q^{-1} & 1 & q & \cdots & q & q^{-2} & 1 & 1 & \cdots & 1 \\
q^{-1} & q^{-1} & 1 & \cdots & q & q^{-2} & q^{-2} & 1 & \ldots & 1 \\
\cdot & . & . & \cdots & \cdot & . & . & . & \cdots & . \\
q^{-1} & q^{-1} & q^{-1} & \ldots & 1 & q^{-2} & q^{-2} & q^{-2} & \ldots & 1 \\
1 & q^{2} & q^{2} & \cdots & q^{2} & 1 & 1 & 1 & \cdots & 1 \\
1 & 1 & q^{2} & \cdots & q^{2} & 1 & 1 & 1 & \cdots & 1 \\
. & . & . & \cdots & . & . & . & . & \cdots & . \\
1 & 1 & 1 & \ldots & 1 & 1 & 1 & 1 & \cdots & 1
\end{array}\right)
$$

and $P\left(\lambda_{\emptyset}\right)$ can be presented by the twisted group algebra $k^{\sigma} P$ by 1.6 , where $P$ has rank $2 n$ and an antisymmetric bimultiplicative map $\sigma$ is given by

$$
\sigma\left(e_{i}, e_{j}\right)=\lambda_{i j}^{1 / 2}
$$

By 2.7, $k P$ is a Poisson algebra with Poisson bracket induced by the antisymmetric bilinear map $u \in Z^{2}(P, k)$ defined by

$$
q^{u\left(e_{i}, e_{j}\right)}=\sigma^{2}\left(e_{i}, e_{j}\right)=\lambda_{i j}
$$

4.2. Let $A_{n}$ be the commutative algebra $A_{n}=k\left[y_{1}, \ldots, y_{n}, x_{1}, \ldots, x_{n}\right]$ with $2 n$ variables and for each $i=1, \ldots, n$, set, as in $\mathbf{3 . 3}$,

$$
z_{i}=q^{-2}\left(1-q^{2}\right) \sum_{1 \leqslant l \leqslant i} q^{l-i} y_{l} x_{l} .
$$


Then we have that

$$
y_{i} x_{i}=q^{2}\left(1-q^{2}\right)^{-1}\left(z_{i}-q^{-1} z_{i-1}\right),\left(z_{0}=0\right), \quad 1 \leqslant i \leqslant n .
$$

Let $C$ be the subalgebra of $A_{n}$ generated by $y_{1}, \ldots, y_{n}, z_{1}, \ldots, z_{n}$ and let $D$ be the localisation of $C$ with respect to the multiplicative set generated by $y_{1}, \ldots, y_{n}, z_{1}, \ldots, z_{n}$. Then $C \subseteq A_{n} \subseteq D \cong k P$ since each $y_{i}$ is invertible in $D$ and so $x_{i} \in D$ for each $i$, and $D$ has a Poisson bracket endowed from the isomorphism from $D$ onto $k P$ defined by

$$
y_{i} \mapsto t_{e_{i}}, z_{i} \mapsto t_{e_{n+i}}
$$

More precisely, $D$ has the following Poisson bracket:

$$
\begin{array}{llll}
\left\{y_{i}, y_{j}\right\}=y_{i} y_{j} & (i<j) & \left\{y_{i}, z_{j}\right\}=0 & (i \leqslant j) \\
\left\{y_{i}, z_{j}\right\}=-2 y_{i} z_{j} & (i>j) & \left\{z_{i}, z_{j}\right\}=0 & (\text { all } i, j) .
\end{array}
$$

Thus $C$ becomes a sub-Poisson-algebra of $D$ and $z_{n}$ is a Poisson central element of $D$. Moreover, $A_{n}$ is also a sub-Poisson-algebra of $D$ because we have the following formulas in $D: \widehat{q}=q^{2}\left(1-q^{2}\right)^{-1}$

$$
\begin{aligned}
& \left\{x_{i}, z_{j}\right\}=\left\{\widehat{q} y_{i}^{-1}\left(z_{i}-q^{-1} z_{i-1}\right), z_{j}\right\}=0 \\
& \left\{x_{i}, z_{j}\right\}=\left\{\widehat{q} y_{i}^{-1}\left(z_{i}-q^{-1} z_{i-1}\right), z_{j}\right\}=2 x_{i} z_{j} \\
& \left\{y_{i}, x_{j}\right\}=\left\{y_{i}, \widehat{q} y_{j}^{-1}\left(z_{j}-q^{-1} z_{j-1}\right)\right\}=-y_{i} x_{j} \\
& \left\{y_{i}, x_{i}\right\}=\left\{y_{i}, \widehat{q} y_{i}^{-1}\left(z_{i}-q^{-1} z_{i-1}\right)\right\}=2 q^{-1} \widehat{q} z_{i-1} \\
& \left\{x_{i}, x_{j}\right\}=\left\{\widehat{q} y_{i}^{-1}\left(z_{i}-q^{-1} z_{i-1}\right), \widehat{q} y_{j}^{-1}\left(z_{j}-q^{-1} z_{j-1}\right)\right\}=-x_{i} x_{j} \quad(i<j) .
\end{aligned}
$$

4.3. We define an admissible set of $A_{n}$ as in 3.4. We shall say that a subset $T$ of $\wp_{n}=\left\{x_{1}, \ldots, x_{n}, y_{1}, \ldots, y_{n}, z_{1}, \ldots, z_{n}\right\}$ is admissible if $T$ satisfies the conditions:

(i) $y_{i} \in T$ or $x_{i} \in T$ if and only if $z_{i} \in T$ and $z_{i-1} \in T, 2 \leqslant i \leqslant n$.

(ii) $y_{1} \in T$ or $x_{1} \in T$ if and only if $z_{1} \in T$.

As in 3.7, if $T$ is an admissible set of $A_{n}$ such that $y_{i} \in T$ and $x_{i} \in T$ for some $i$ then the index $i$ is said to be removable.

LEMMA 4.4.

(1) Every ideal generated by an admissible set of $A_{n}$ is prime Poisson.

(2) For each prime Poisson ideal $P$ of $A_{n}, P \cap \wp_{n}$ is an admissible set of $A_{n}$.

(3) If $T$ is an admissible set of $A_{n}$ with removable indices then there are $m<n$ and an admissible set $T^{\prime}$ of $A_{m}$ such that $A_{n} /\langle T\rangle \cong A_{m} /\left\langle T^{\prime}\right\rangle$ as Poisson algebras and $T^{\prime}$ has no removable indices. 
Proof: Note that each $z_{i}$ is an irreducible element of $B .(1)$ and (2) follow immediately from 4.2, and (3) follows from the modified proof of $\mathbf{3 . 8}$.

4.5. Let $T$ be an admissible set of $A_{n}$. Define $S_{T}$, as in the proof of $\mathbf{3 . 6}$, by

$$
\begin{aligned}
& T_{y}=\left\{y_{j} \mid y_{j} \notin T, x_{j} \in T\right\} \\
& T_{x}=\left\{x_{j} \mid x_{j} \notin T, y_{j} \in T\right\} \\
& S_{T}=\left\{y_{j}, z_{j} \mid z_{j} \notin T\right\} \cup\left\{y_{j} \mid z_{j} \in T, y_{j} \notin T, x_{j} \notin T\right\} \cup T_{y} \cup T_{x}
\end{aligned}
$$

and let $C_{T}$ be the subalgebra of $A_{n}$ generated by $S_{T}$. Then $C_{T}$ is embedded into $A_{n} /\langle T\rangle$, the localisation $D_{T}$ of $C_{T}$ with respect to the multiplicative set generated by $S_{T}$ is isomorphic to a group algebra $k P^{\prime}$ and

$$
C_{T} \subseteq A_{n} /\langle T\rangle \subseteq D_{T} \cong k P^{\prime} .
$$

The commutative algebra $D_{T} \cong k P^{\prime}$ has the Poisson bracket induced by an antisymmetric bilinear map $u$ defined by

$$
q^{u\left(e_{i}, e_{j}\right)}=\sigma^{2}\left(e_{i}, e_{j}\right)=\lambda_{i j}
$$

where $\sigma^{2}\left(e_{i}, e_{j}\right)=\lambda_{i j}$ is the $(i, j)$-entry of the defining matrix $\lambda_{T}$ in the twisted group algebra $P\left(\lambda_{T}\right)$ of $\mathbf{3 . 6}$.

LEMmA . The Poisson structures of $A_{n} /\langle T\rangle$ induced by that of $A_{n}$ and by that of $D_{T} \cong k P^{\prime}$ are equal and $C_{T}$ is a sub-Poisson algebra.

Proof: Straightfoward.

Theorem 4.6. For each admissible set $T$ of the Poisson algebra $A_{n}$, let $\operatorname{Symp}_{T}\left(A_{n}\right)$ be the set of all symplectic ideals $Q$ of $B$ with $Q \cap \wp_{n}=T$. Then there is a bijection between $\operatorname{Symp}_{T}\left(A_{n}\right)$ and $\operatorname{Max}\left(k\left[t_{1}^{ \pm 1}, \ldots, t_{s}^{ \pm 1}\right]\right)$, and $\operatorname{Symp}\left(A_{n}\right)=$ $\bigsqcup_{T} \operatorname{Symp}_{T}\left(A_{n}\right)$, where $s=\operatorname{deg}(T)$ when $T$ is considered as an admissible set of $\mathcal{O}_{q}^{n}$. Moreover, there is a bijection between $\operatorname{Prim} \mathcal{O}_{q}^{n}$ and $\operatorname{symp}\left(A_{n}\right)$.

Proof: If $n=1$ then $A_{1}$ has trivial Poisson structure and so there is nothing to prove since symplectic ideals of Poisson algebra with trivial Poisson structure are only maximal ideals. Assume $n>1$. By induction on $n$, and 4.4 (3), we may assume that $T$ has no removable indices. By 4.5 and 2.7, the centre of the twisted group algebra $P\left(\lambda_{T}\right)$ of 3.6 and the Poisson centre of $D_{T}$ of 4.5 are equal, hence the conclusion follows immediately from 2.6, 3.11 and 3.14.

Theorem 4.7. All symplectic ideals of the Poisson algebra $A_{n+1} / I$, $I=\left\langle y_{1}-q^{1 / 2}(1+q)^{-1} x_{1}\right\rangle$, correspond bijectively to Prim $\mathcal{O}_{q}\left(a k^{2 n+1}\right)$.

Proof: By $[9,5]$, the map $f$ from $\mathcal{O}_{q}^{n+1}$ into $\mathcal{O}_{q}\left(o k^{2 n+1}\right)$ given by

$$
y_{1} \mapsto q^{1 / 2}(1+q)^{-1} z_{0}, x_{1} \mapsto z_{0}, y_{i} \mapsto y_{i-1}, x_{i} \mapsto x_{i-1} \quad(i \geqslant 2)
$$


is an epimorphism with kernel $\left\langle y_{1}-q^{1 / 2}(1+q)^{-1} x_{1}\right\rangle$, and the ideal $I=\left\langle y_{1}-\right.$ $\left.q^{1 / 2}(1+q)^{-1} x_{1}\right\rangle$ of $A_{n+1}$ is a prime Poisson ideal and thus $A_{n+1} / I$ is a Poisson algebra. Moreover, in 4.6, all primitive ideals of $\mathcal{O}_{q}^{n+1}$ containing $\left\langle y_{1}-q^{1 / 2}(1+q)^{-1} x_{1}\right\rangle$ correspond bijectively to all symplectic ideals of $A_{n+1}$ containing $I$.

\title{
REFERENCES
}

[1] M. Akhavizadegan and D.A. Jordan, 'Prime ideals of quantized Weyl algebras' (to appear).

[2] K.R. Goodearl and T.H. Lenagan, 'Catenarity in quantum algebras', J. Pure Appl. Algebra 111 (1996), 123-142.

[3] K.R. Goodearl and E.S. Letzter, 'Prime factor algebras of the coordinate ring of quantum matrices', Proc. Amer. Math. Soc. 121 (1994), 1017-1025.

[4] D.A. Jordan, 'A simple localization of the quantized Weyl algebra', J. Algebra 174 (1995), 267-288.

[5] A. Joseph, Quantum groups and their primitive ideals, A series of modern surveys in mathematics, (3. Folge-Band 29) (Springer-Verlag, Berlin, Heidelberg, New York, 1995).

[6] J.C. McConnell and J.J. Pettit, 'Crossed products and multiplicative analogues of Weyl algebras', J. London Math. Soc. 38 (1988), 47-55.

[7] J.C. McConnell and J.C. Robson, Noncommutative Noetherian rings (Wiley-Interscience, New York, 1987).

[8] Sei-Qwon Oh, 'Primitive ideals of the coordinate ring of quantum symplectic space', $J$. Algebra 174 (1995), 531-552.

[9] Sei-Qwon Oh, 'Catenarity in a class of iterated skew polynomial rings', Comm. Algebra 251 (1997), 37-49.

[10] S.P. Smith, 'Quantum groups: An introduction and survey for ring theorists', in Noncommutative Rings, (S. Montgomery and L. Small, Editors), M.S.R.I. Publ. 24 (Springer-Verlag, Berlin, Heidelberg, New York, 1992), pp. 131-178.

[11] M. Takeuchi, 'Matric bialgebras and quantum groups', Israel J. Math. 72 (1990), 232-251.

\author{
Department of Mathematics \\ Chungnam National University \\ Taejon 305-764 \\ Korea \\ e-mail: sqoh@math.chungnam.ac.kr \\ cgpark@math.chungnam.ac.kr
}

